\title{
Caracterización de Propiedades Fisicoquímicas, Textura y Calidad Microbiológica de Butifarra Comercializada en Cartagena (Colombia)
}

\author{
Diofanor Acevedo*, Clemente Granados, Piedad M. Montero \\ Universidad de Cartagena, Facultad de Ingeniería, Departamento de Ingeniería de Alimentos, Avenida el \\ Consulado, Calle 30 No. 48-152. Cartagena, Bolívar-Colombia \\ (e-mail: diofanor3000@gmail.com) \\ * autor a quien debe ser dirigida la correspondencia
}

Recibido Mar. 11, 2014; Aceptado Jun. 6, 2014; Versión final recibida Jul. 8, 2014

\begin{abstract}
Resumen
El objetivo del presente estudio fue caracterizar las propiedades fisicoquímicas, textura y calidad microbiológica de butifarra comercializada en la calle y en supermercados en la ciudad de Cartagena (Colombia). La venta de alimentos callejeros en América Latina y El Caribe constituye un fenómeno que reviste gran importancia sociocultural, económica y sanitaria para la región, lo que amerita un estudio como el que se presenta. Se realizaron análisis proximales, microbiológicos y texturales a 30 muestras provenientes de supermercados, Mercado de Bazurto y venta callejera, ubicados en la ciudad de Cartagena. Solo las butifarras vendidas en supermercado cumplen con la calidad premium de acuerdo a su contenido fisicoquímico, y además son las únicas que cumplen con los requisitos microbiológicos expuestos en la Norma Técnica Colombiana. Las butifarras evaluadas poseen un perfil de textura similar a los reportados por otros autores para este tipo de productos.
\end{abstract}

\section{Characterization of physicochemical properties, texture and microbiological quality of sausage sold in Cartagena (Colombia)}

\begin{abstract}
The objective of this study was to characterize the physicochemical properties, texture and microbiological quality of sausages sold in the city of Cartagena (Colombia). The street food vending in Latin America and the Caribbean is a phenomenon of great sociocultural, economic and health importance in the region, so the subject deserves a study like the one presented ion this paper. Proximal, microbiological and textural analysis of 30 samples from supermarkets Bazurto market and street vendors located in the city of Cartagena were performed. The sausages sold in supermarkets only meet the premium quality according to their physico-chemical content, and they are the only ones that meet the microbiological criteria set by the Colombian Technical Standard. The sausages evaluated have a profile similar to those reported by other authors for this type of product texture.
\end{abstract}




\section{INTRODUCCIÓN}

La butifarra fresca es un producto cárnico procesado homogenizado, cocido, embutido en tripa comestible, elaborado a base de carne de res y cerdo, adicionado de sal, grasa y especias. De sabor picante que puede contener o no sales de curación, mantenido el color pálido característico y no contiene colorantes (NTC 1325, 2008). Las enfermedades transmitidas por alimentos (ETA) han sido consideradas como un grave problema de salud pública a escala mundial, donde los alimentos se reconocen como el vector principal de las enfermedades entéricas agudas (Jiménez et al., 2012). En el continente americano las ETA figuran entre las primeras cinco causas de muerte en los menores de 5 años, con una incidencia promedio anual de cuatro episodios diarreicos anuales por niño. Cada año aumenta el número de personas afectadas por ETA, causadas por la ingestión de alimentos mal procesados o preparados (De Curtis et al., 2000). A nivel mundial los productos cárnicos ocupan un lugar relevante como vehículo de agentes microbianos causantes de enfermedades transmitidas por alimentos (ETA), de allí que el estudio de la calidad microbiológica de los productos cárnicos procesados sea de gran interés en la industria alimentaria. Muchas investigaciones se han elaborado referente al tema (Wang et al., 2013). Ambrosiadis et al., (2004) realizaron análisis físicoquímicos, microbiológicos y sensoriales a 67 muestras de embutidos tradicionales griegos, mientras que Fernández-López et al., (2008) estudiaron los perfiles físico-químicas y microbiológicas de "salchichón" (salchicha fermentada seca español) enriquecidos con fibra de naranja. Por otro lado, Izquierdo et al., (2007) evaluaron las características físico-químicas, microbiológicas y calidad sensorial de salchichas elaboradas con mezclas de Cachama negra (Colossoma macropomum) y carne de res.

La venta de alimentos callejeros en América Latina y El Caribe constituye un fenómeno que reviste gran importancia sociocultural, económica y sanitaria para la región. Este tipo de venta va aumentado debido a diversas causas socioeconómicas entre las que se pueden mencionar: el deterioro de las condiciones de vida en las áreas rurales; la migración rural-urbana; el proceso de urbanización intensiva; el fenómeno de los desplazados por la violencia, la guerrilla y el narcotráfico; el proceso de ajuste y recesión económica que han provocado desempleo, deterioro en el poder adquisitivo de la población, desplazamiento de poblaciones a zonas alejadas de su sitio de trabajo y deterioro de los servicios de salud, educación y vivienda (Romero et al., 2013).

En Colombia la fabricación de productos cárnicos como la butifarra no ha sido estandarizada y se monitorizan en general subjetivamente y sin un control estricto de las características requeridas para estos productos. Sin embargo, si se mantienen las condiciones de higiene y las propiedades intrínsecas de los alimentos, los embutidos tradicionales podrían ser considerados productos seguros (Romero et al., 2013). A pesar de la popularidad de la butifarra en la Costa Atlántica Colombiana, especialmente en Cartagena, donde su principal venta es la callejera, son nulos los estudios encontrados sobre esta. Debido a que la información científica sobre butifarra es escasa y considerando el interés social y comercial de la caracterización de productos típicos y tradicionales en Latinoamérica, el objetivo de este estudio fue caracterizar las propiedades fisicoquímicas, textura y la calidad microbiológica de butifarra comercializada en Cartagena (Colombia).

\section{MATERIALES Y MÉTODOS}

\section{Muestras}

Se tomaron 10 muestras de diferentes tipos de butifarra teniendo en cuenta el tipo de comercialización. Estas se escogieron al azar de supermercados, Mercado de Bazurto y venta callejera, ubicados en la ciudad de Cartagena (Colombia), obteniendo un total de 30 muestras. Las mismas fueron empacadas en bolsas de polietileno de primer uso debidamente rotuladas para luego ser refrigeradas.

\section{Análisis de composición proximal y microbiológica}

De acuerdo a lo indicado en la Norma Técnica Colombiana 1325 (2008) se determinó contenido de humedad (NTC 1663), materia grasa (NTC 1662), proteína (NTC 1556), Almidón (NTC 4566), recuento total aerobios (NTC 4519), coliformes totales y fecales (NTC 4458), S. aureus (NTC 4779) y Salmonella (NTC 4574). El conteo de microorganismos fue en Log de UFC/g, a excepción de la Salmonella que se expresó en Salmonella/25 g. Los resultados se muestran como promedios de los datos obtenidos para cada sitio de muestreo.

\section{Análisis del perfil de textura (TPA)}

Se utilizó un texturómetro Marca Shimadzu y se realizó la determinación del perfil de textura por medio del Rheometer software. Para esto se cortaron rodajas de butifarra de aproximadamente $1,5 \mathrm{~cm}$ de grosor y se 
dejaron reposar por una hora a temperatura ambiente dentro de una bolsa de polietileno para evitar la pérdida de humedad (Granados et al., 2013; Isaza et al., 2010). El método de TPA fue realizado mediante la aplicación de una fuerza de compresión dos veces sucesivas en las muestras, con el fin de simular la masticación humana, obteniendo la curva fuerza/tiempo y calculados los parámetros: cohesividad, dureza, gomosidad y elasticidad.

\section{Análisis de datos}

En la investigación, se manejó un diseño experimental totalmente aleatorio. Las determinaciones se efectuaron por triplicado y los resultados expresados como la media con su desviación estándar. Se utilizó el programa GraphPadlnstats versión 3.1 y Minitab 16 Statistical Software para el análisis de los datos, se aplicó un análisis de varianza (ANOVA) y el análisis de medias, mediante un test de HSD Tukey, se fijó el nivel de significancia a $p \leq 0,05$.

\section{RESULTADOS Y DISCUSIÓN}

\section{Análisis Proximal}

Los resultados del análisis proximal de las butifarras recolectadas en diferentes zonas de expendio se aprecian en la Tabla 1. En términos generales los diferentes parámetros de la composición proximal de las butifarras analizadas en esta investigación, no mostraron diferencias amplias entre una y otra. Las únicas butifarras diferentes en cuanto a humedad y proteínas fueron las procedentes de supermercado $(p \leq 0,05)$. Referente a la composición de grasa y almidón todas las butifarras tuvieron diferencias estadísticamente significativas $(p \leq 0,05)$. Como se observa en la Tabla 1 las únicas butifarras que cumplen con los requisitos para ser consideradas un producto Premium de acuerdo a la NTC 1325 (2008) son las provenientes de supermercado, ya que las provenientes de ventas callejera y de mercado no alcanzan el umbral de contenido proteico que requiere la Norma.

Tabla 1: Composición proximal (\%) de butifarras analizadas

\begin{tabular}{|c|c|c|c|c|}
\hline Componente & Mercado Bazurto & Venta Callejera & Supermercado & Permitido Premium \\
\hline Humedad & $65,91 \mp 1,22^{\mathrm{a}}$ & $65,3 \mp 1,03^{\mathrm{a}}$ & $64 \mp 1,33^{\mathrm{b}}$ & Máximo 86 \\
\hline Proteínas & $13,57 \mp 0,84^{\mathrm{a}}$ & $12 \mp 0,85^{\mathrm{a}}$ & $17 \mp 0,76^{\mathrm{b}}$ & Mínimo 14 \\
\hline Grasas & $14 \mp 0,54^{\mathrm{a}}$ & $15 \mp 0,64^{\mathrm{b}}$ & $13 \mp 0,74^{\mathrm{c}}$ & Máximo 28 \\
\hline Almidón & $3,12 \mp 0,00^{\mathrm{a}}$ & $4 \mp 0,074^{\mathrm{b}}$ & $2,39 \mp 0,02^{\mathrm{c}}$ & Máximo 3 \\
\hline
\end{tabular}

El menor nivel de proteínas en las butifarras de venta callejera y de mercado, puede ser explicado por el hecho de que su formulación incluye alta porcentualidad de harinas, que incrementa la presencia de carbohidratos, disminuyendo así la cantidad final de proteínas (Márquez et al., 2008). Tanto los porcentajes de proteína como los de grasa dan una idea clara de que las butifarras de supermercado son elaboradas con mayor relación carne/grasa en contraste con las elaboradas de Mercado y venta callejera. La cantidad de proteína influye en la calidad de las butifarras, debido a que ésta permite mejores propiedades funcionales de ligado de la grasa, sin dejar de mencionar la importancia que tiene sobre el aspecto nutritivo (González-Tenorio et al., 2013).El contenido de proteína fue similar pero menor a los reportados por Márquez et al., (2008) para butifarras elaboradas a partir de cachama negra.

En cuanto al contenido de humedad, son similares al contenido de tilapia ahumada $(67,96 \%)$ de Yanar y Erhan (2006), así como también para salchichas de pavo (62-67\%) de Beggs et al., (1995) y con los reportados por Yang et al., (2007) para salchichas de cerdo bajas en grasa con harina de avena (67-68\%). El porcentaje de grasa fue mayor en las butifarras de venta callejera, y menor en los de venta de supermercado. En la calidad de las butifarras, la grasa es una propiedad con repercusiones importantes debido a que está involucrada directamente el sabor, textura, valor nutritivo y estabilidad oxidativa (González-Tenorio et al., 2013).El contenido en cenizas en las butifarras analizadas fue muy cercano, pero aun así fue mayor en ventas callejeras y mercado. Estos contenidos se pueden atribuir a la mayor adición de sales (principalmente $\mathrm{NaCl}$ ) en la elaboración de este tipo de productos (González-Tenorio et al., 2013).

Los resultados proximales de este estudio son muy similares a los reportados por Hleap y Velasco (2010; 2012). En ambos trabajos estos investigadores evaluaron los parámetros fisicoquímicos durante el almacenamiento de salchichas elaboradas a partir de tilapia roja (Oreochromis $s p$.). Por otro lado, En estudios anteriores Márquez et al., (2008) elaboraron semiconservas (hamburguesa, butifarra y chorizo) a partir de pulpa de cachama negra (Colossoma macropomum) obtuvieron valores proximales un poco diferentes que los de esta investigación: Humedad $(74 \%)$, proteína $(18,2)$, grasa $(1,5)$, cenizas $(6,1)$ y 
carbohidratos $(0,2)$. Los resultados proximales de esta investigación difieren a los obtenidos por otros autores en productos cárnicos a base de materias primas similares (Gonzales-Tenorio et al., 2013; Pérez et al., 1999). Estas diferencias pueden ser debidas al método, formulación y producto usados (Tirado et al., 2013).

\section{Análisis microbiológico}

En la Tabla 2 se pueden observar los resultados de recuento total de Aerobios, Coliformes totales y fecales, S. aureus y Salmonella expresados como Log de UFC/g que se realizaron a las muestras de butifarras. Según la Tabla las butifarras de Supermercado son los únicos que se encuentran dentro de todos los rangos estipulados por la Norma Técnica Colombiana 1325 (2008) para índice máximo permisible para nivel de buena calidad en productos cárnicos procesados. Los malos resultados de las demás butifarras se pueden explicar debido al mal almacenamiento del producto o a la falta de buenas prácticas de manufactura durante la elaboración del producto en las centrales de abasto y la calle. Estudios realizados en América Latina, han revelado que un gran porcentaje de vendedores ambulantes no cuentan con un sistema de abastecimiento de agua con calidad sanitaria y cantidades suficiente para las necesidades diarias. Esto ocasiona deficientes lavado de equipos y utensilios, así como lavado de manos, situación que la convierte en fuente de contaminación al facilitar la proliferación de microorganismos. Desde el punto de vista sanitario, la venta de alimentos en la vía pública en ocasiones es controvertida. Lo anterior debido a las deficientes prácticas de higiene en la preparación de los alimentos, las cuales tienden a presentar riesgos considerables para la salud por exponerse a vertimientos de residuales líquidos, sólidos, presencia de vectores y ambientes contaminados (Romero et al., 2013).

Tabla 2: Análisis microbiológico de butifarras analizadas

\begin{tabular}{|c|c|c|c|c|c|}
\hline Lugar muestreo & $\begin{array}{c}\text { Aerobios } \\
\text { (UFC/g) }\end{array}$ & $\begin{array}{c}\text { C. totales } \\
\text { (UFC/g) }\end{array}$ & $\begin{array}{c}\text { C. fecales } \\
\text { (UFC/g) }\end{array}$ & $\begin{array}{c}\text { S. aureus } \\
\text { (UFC/g) }\end{array}$ & Salmonella/25g \\
\hline Mercado de Bazurto & 19,10 & 15,71 & 8,5 & 2 & 0 \\
\hline Venta callejera & 35,79 & 19,4 & 13,3 & 5 & 0 \\
\hline Supermercado & 5 & 0 & 0 & 0 & 0 \\
\hline Permitido & 5 & 2 & 2 & $<2$ & 0 \\
\hline
\end{tabular}

\section{Análisis de perfil de Textura (TPA)}

Los resultados de TPA para las butifarras se muestran en la Tabla 3. Dependiendo de su procedencia todas las butifarras presentaron diferencias significativas en sus parámetros texturales $(p \leq 0,05)$. En relación a las variables de textura evaluadas a través del TPA, no se encontraron referencias bibliográficas para butifarras, lo que hace que estos resultados sean novedosos. En cuanto a la textura de butifarras, la materia prima influye contundentemente (la cantidad de carne, grasa, tejido conjuntivo), la eventual presencia de almidones o proteínas no cárnicas, el diámetro del embutido y el grado de secado. El papel de la textura en la calidad de butifarras no es del todo claro y depende de la variedad de butifarra de que se trate. Las diferencias en las muestras analizadas para cada tipo se podrían atribuir a variaciones en los factores anteriormente mencionados, siendo probable que la humedad sea uno de los más implicados (GonzálezTenorio et al., 2013).

Las butifarras de supermercado fueron las más duras. Este tipo de butifarra presentó casi el doble de dureza respecto a las butifarras de venta callejera, y casi cinco unidades más duras que las del Mercado. Esta diferencia está relacionada con el menor contenido en humedad de butifarras de supermercado (González-Tenorio et al., 2013). Aun así las butifarras de supermercado no están por encima de datos reportados por otros autores (Granados et al., 2013; Hleap y Velasco, 2010; Herrero, 2008) en diferentes productos cárnicos embutidos.

Tabla 3: Análisis de perfil de textura a butifarras analizadas

\begin{tabular}{|c|c|c|c|}
\hline & Mercado de Bazurto & Venta Callejera & Supermercado \\
\hline Dureza(N) & $20,34 \mp 0,56^{\mathrm{a}}$ & $15,56 \mp 0,67^{\mathrm{b}}$ & $24,62 \mp 0,87^{\mathrm{c}}$ \\
\hline Adhesividad(N) & $-0,56 \mp 0,04^{\mathrm{a}}$ & $-0,34 \mp 0,03^{\mathrm{b}}$ & $-0,62 \mp 0,06^{\mathrm{c}}$ \\
\hline Cohesividad (Adimencional) & $0,61 \mp 0,07^{\mathrm{a}}$ & $0,52 \mp 0,01^{\mathrm{b}}$ & $0,70 \mp 0,02^{\mathrm{c}}$ \\
\hline Elasticidad(Adimencional) & $0,45 \mp 0,06^{\mathrm{a}}$ & $0,350 \mp 0,054^{\mathrm{b}}$ & $0,6 \mp 0,01^{\mathrm{c}}$ \\
\hline Masticabilidad(N) & $1,08 \mp 0,09^{\mathrm{a}}$ & $0,78 \mp 0,065^{\mathrm{b}}$ & $1,54 \mp 0,01^{\mathrm{c}}$ \\
\hline
\end{tabular}


La mayor adhesividad la presentaron las butifarras de supermercado, y las menores las de venta callejera. En cuanto a la adhesividad, es importante resaltar que los valores obtenidos son negativos, lo cual indica que la textura de las butifarras es pegajosa o adhesiva, es decir, cuando el producto es consumido éste se adhiere al paladar, lo que conlleva a realizar un trabajo necesario para retirarlo. Esto puede deberse a la menor cantidad de carbohidratos en la formulación (González-Tenorio et al., 2013). Los valores de cohesividad son similares a los reportados por Granados et al., (2013), pero mayores a los reportados por otros autores (Herrero, 2008; Hleap y Velasco, 2010). Los valores de elasticidad y masticabilidad por su parte son menores a las reportadas por Hleap y Velasco (2010). Probablemente estas diferencias se deban a que el atún, ingrediente cárnico utilizado en la elaboración de las salchichas en el trabajo de Hleap y Velasco (2010), se caracteriza por una elevada capacidad de retención de agua y valores altos en el contenido de humedad (García et al., 2005).

\section{CONCLUSIONES}

Dependiendo la procedencia así es la composición, textura y calidad microbiológica de la butifarra. La comercializada en supermercado fue la que presentó mayor contenido de proteína y menor de carbohidrato. Estas diferencias son debidas a las desiguales cantidades de carne y grasa utilizadas, y diferencias de carbohidratos adicionadas a la formulación para cada producto, siendo este mayor en los productos de venta libre. En cuanto a los análisis microbiológicos la butifarra comercializada en supermercado fue la única que se encontró dentro del rango de permisibilidad para consumo en Colombia según la NTC 1325 (2008).

\section{REFERENCIAS}

Ambrosiadis, J. y otros tres autores, Physicochemical, microbiological and sensory attributes for the characterization of Greek traditional sausages, Meat Science: 66(2), 279-287 (2004).

Beggs, K.L.H., J.A. Bowers, and D. Brown, Sensory and physical characteristics of reduced fat turkey frankfurters with modified corn starch and water, J. Food Science: 62(6), 1240-1244 (1995)

De Curtis, M.L., O. Franceschi y N. De Castro, Determinación de la calidad microbiológica de alimentos servidos en comedores de empresas privadas. ALAN: 50(2), 177-182 (2000)

Fernández-López, J. y otros tres autores, Physico-chemical and microbiological profiles of "salchichón" (Spanish dry-fermented sausage) enriched with orange fiber, Meat Science: 80(2), 410-417 (2008).

García, A. y otros cinco autores, Formulación de salchichas con atún y carne: vida útil y aceptabilidad, Revista científica, FCV-LUZ, 15(3), 272-278 (2005)

Gonzales-Tenorio, R. y otros tres autores, Caracterización de Propiedades Químicas y Fisicoquímicas de Chorizos Comercializados en la Zona Centro de México, Información Tecnológica: 24(2), 3-14 (2013)

Granados, C., L.E. Guzmán y D. Acevedo, Análisis proximal, sensorial y de textura de salchichas elaboradas con subproductos de la industria procesadora de atún (Scombridae thunnus), Información tecnológica, 24(6), 29-34 (2013)

Herrero, A.M. y otros cinco autores, Tensile properties of cooked meat sausages and their correlation with texture profile analysis (TPA) parameters and physico-chemical characteristics, Meat Science: 80(3), 690$696(2008)$

Hleap, J. y A.V. Velasco, Análisis de las propiedades de textura durante el almacenamiento de salchichas elaboradas a partir de Tilapia roja (Oreochromis $s p$.), Revista de la Facultad de Ciencias Agropecuarias: 8(2), 46-56 (2010)

Hleap, J. y A.V. Velasco, Parámetros fisicoquímicos durante el almacenamiento de salchichas elaboradas a partir de tilapia roja (Oreochromis sp.), Revista de la Facultad de Ciencias Agropecuarias: 10(1), 42-50 (2012)

Isaza, J. y otros cuatro autores, Producción y propiedades funcionales de plasma bovino hidratado en embutido tipo salchichón, Revista Colombiana de Ciencias Pecuarias: 23(2), 199-206 (2010) 
Izquierdo, P. y otros cuatro autores, Proximal, Microbiológico y Evaluación Sensorial de Salchichas Elaboradas a Base de Cachama Negra (Colossoma macropomum), Revista Científica: 17(3), 294-300 (2007)

Jiménez, M., C. Chaidez y J. León, Calidad microbiológica de carne de res comercializada en el mercado municipal de Culiacán, Sinaloa, Veterinaria México: 43(4), 273-284 (2012)

Marquez, V.M. D. Cabrera y H. Rico, Elaboración de semiconservas (hamburguesa, butifarra y chorizo) a partir de cachama negra (Colossoma macropomum), Revista RETAKVN: 1(1), 110-123 (2008)

NTC 1325, Instituto Colombiano de Normas Técnicas y Certificación (Colombia), Establece los requisitos y los métodos de ensayo que deben cumplir productos cárnicos procesados no enlatados. Quinta Actualización, Bogotá, Colombia (2008)

Pérez, L., y otros 4 autores., Evaluación química y sensorial del chorizo tipo Pamplona, elaborado a partir de carne de Cerdo Pelón Mexicano y de Cerdo Mejorado, Veterinaria México, 30 (1) 33-40 (1999).

Tirado, D.F., D. Acevedo y L.E. Guzmán, Coeficientes convectivos de transferencia de calor durante el freído de láminas de tilapia "Oreochromis niloticus", Información Tecnológica: 24(6), 41-46 (2013)

Wang, X.H. y otros cuatro autores, Effects of inoculating Lactobacillus sakei starter cultures on the microbiological quality and nitrite depletion of Chinese fermented sausages, Food Control: 32(2), 591-596 (2013)

Yanar, Y. y M.C. Erhan, Effects of brine concentration on shelf-life of hot-smoked tilapia (Oreochromis niloticus) stored at $4^{\circ} \mathrm{C}$, Food Chemistry: 97, 244-247 (2006)

Yang, H.S. y otrostresautores, Textural and sensory properties of low fat pork sausages with added hydrated oatmeal and tofu as texture-modifying agents, Meat Science: 75, 283-289 (2007) 